\title{
The Use of Magnetic Seeds and Radiofrequency Identifier Tags in Breast Surgery for Non-palpable Lesions
}

\author{
SALIM TAYEH, RITIKA GERA, NICHOLAS PERRY, MICHAEL MICHELL, \\ ANMOL MALHOTRA and KEFAH MOKBEL
}

The London Breast Institute, The Princess Grace Hospital, London, U.K.

\begin{abstract}
Background: Wire-guided localisation (WGL) remains the most widely used technique to guide surgical excision of non-palpable breast lesions worldwide. However, recent technological advances have led to the advent of less invasive radiation-free localisation methods to overcome the limitations of WGL. Patients and Methods: This study prospectively evaluated the role of two radiation-free nonwire localisation methods. Magnetic seeds $(n=16)$ and radiofrequency tags $(n=6)$ were deployed under imaging guidance to guide the surgical excision in 19 consecutive patients. Results: The identification/retrieval and migration rates were $100 \%$ and $4.5 \%$, respectively. Twenty-one out of $22(95.5 \%)$ cases had clear surgical margins and no complications were observed. All radiologists and the surgeon rated these methods as being much better than wire localisation. Patient satisfaction data were recorded using a linear visual analogue scale $(n=10 / 19)$. The mean score was 9.7/10 (range=8-10). Conclusion: Our study provides further evidence that radiation-free wireless breast localisation is an effective alternative to WGL.
\end{abstract}

The number of patients with non-palpable breast lesions has increased due to the widespread improvement and use of screening mammography (1). The advent of vacuum-assisted tissue sampling has led to a reduction in the number of patients requiring open diagnostic surgical excision for indeterminate radiological abnormalities (2). However, in addition to patients with non-palpable breast cancer at initial diagnosis or after neoadjuvant chemotherapy (NACT), open surgical excision is still required in a significant number of patients for accurate

Correspondence to: Mr. Salim Tayeh, Senior Clinical Fellow in Breast and General Surgery, The London Breast Institute, The Princess Grace Hospital, 42-52 Nottingham Place, London, W1U 5NY, U.K. Tel: +44 2079082040, Fax: +44 2079082275, Mob: +44 7867895385, e-mail: salimtayeh@hotmail.co.uk

Key Words: Magnetic seeds, radiofrequency identification tags, nonwire localisation. diagnosis. Therefore, accurate preoperative localisation of these lesions is essential to guide precise surgical excision.

In addition to traditional wire-guided localisation (WGL) devices, there are now many alternatives, such as: Radioactive seed localisation (RSL) (3), a reflector that uses micro impulse radar (4) (SAVI SCOUT; Cianna Medical Inc, Aliso Viejo, CA, USA), magnetic markers (5) (Magseed; Endomagnetics Inc, Austin, TX, USA), and radiofrequency identification (RFID) tags (6) (LOCalizer, Hologic, Santa Carla, CA, USA).

Although on a global scale WGL is currently the most widely used technique, the technique has several disadvantages: In addition to inflexible scheduling with the wire insertion being usually performed on the day of surgery thus affecting the radiology suite and operating theatre efficiency, the protruding wire can be bothersome for the patient, causing discomfort and anxiety, and has the risk of dislodgement, the wire can be transected or displaced during surgery leading to fragment retention, the insertion of the radiological wire can be challenging in patients with very dense breasts, and there are limitations in surgical incision and dissection route with a potential impact on cosmesis and potential injury to other organs such as the pleura causing pneumothorax (7). Another potential limitation of WGL that is under-reported in the literature is the risk of needle stick injury to surgical staff caused by the sharp end of the wire.

Therefore, other alternatives have been evolving to overcome these limitations. Since Gray and colleagues first described RSL as an alternative to WGL in 2001 (3), several studies have compared RSL with wire localisation and demonstrated that the former is a safe and effective alternative. RSL can be carried out entirely within the breast at any time within 5 days of surgery. However, handling radioactive materials requires special licensing and carries strict regulatory requirements, with implications for costeffectiveness and staff training (6).

Non-radioactive non-wire devices are passive and contain no energy source. The Magseed is a nonradioactive metal marker which contains iron particles. The dedicated Sentimag probe generates an alternating magnetic field that transiently magnetizes the iron in the Magseed. The tiny 
magnetic signature generated by the Magseed is then detected by the Sentimag probe. The Magseed device is 5 $\mathrm{mm}$ in length and can be deployed under mammography, ultrasound, or computed tomographic guidance using a preloaded sterile 18-gauge needle and can be detected within $4 \mathrm{~cm}$ depth from the skin surface (8). An RFID tag can communicate a range of information from one serial number to several pages of data. It contains a ferrite rod wrapped with copper and a microprocessor. The $10.5 \mathrm{~mm}$ LOCalizer RFID tag can be deployed through a preloaded sterile 12gauge needle. When the patient is in the supine surgical position, the tag can be detected within $6 \mathrm{~cm}$ depth of the handheld loop probe at the skin surface and $4 \mathrm{~cm}$ depth of the intraoperative surgical dedicated pencil probe $(6,8)$. The Scout device is $12 \mathrm{~mm}$ in size and can be deployed via a 16gauge needle and be detected by radar to $4.5 \mathrm{~cm}$ depth. Its technical design makes it resistant to migration.

The aim of this prospective evaluation was to explore the use of magnetic seeds and RFID tags as non-inferior wireless and radiation-free alternatives to standard WGL.

\section{Patients and Methods}

Patients. Female patients (aged 18 or older) with non-palpable breast lesions requiring surgical excision were enrolled in this study. The indications for surgery included screen-detected non-palpable malignancy, malignant lesions rendered non-palpable post NACT, and pathologically intermediate (B3) non-palpable lesions which required diagnostic surgical excision. One patient with screendetected breast cancer also requested the surgical excision of a small fibroadenoma deeply located in the contralateral breast. This was an observational clinical evaluation; hence formal ethical approval was not required. However, the use of these technologies was approved by the Multidisciplinary Breast Cancer Board of the London Breast Institute and subsequently by the clinical governance team of The Princess Grace Hospital. All participants give their informed written consent. Detailed patient information leaflets regarding both techniques were made available.

Procedure. Magseeds (Endomagnetics) and RFID tags (LOCalizer, Hologic) were investigated. Image-guided deployment was performed up to 30 days prior to surgery to guide the surgical excision of nonpalpable breast lesions. After deployment of Magseed/RFID tag, its position was checked using a post procedure mammogram.

Following informed written consent, 22 lesions in 19 patients were operated upon consecutively by the same surgeon. Sixteen cases employed Magseed technology and six cases utilised RFID tag technology with LOCalizer. Magseeds and RFID tags were deployed in an image-guided manner, for which 21 cases were deployed under ultrasound guidance and one case was deployed under stereotactic digital mammography guidance. Ultrasoundguided deployment was performed in the supine position under local anaesthesia $(\mathrm{n}=21)$. One patient with ductal carcinoma in situ (DCIS) underwent stereotactic Magseed deployment. Stereotactic imaging was performed with the patient lying left in a lateral decubitus position and the Magseed was inserted using a lateralmedial approach.
Surgery was performed within 30 days of deployment. Following identification and surgical excision, the magnetic or RFID probe was used to confirm retrieval and to assess the distance between the Magseed (relative signal intensity) or RFID tag (distance in $\mathrm{mm}$ displayed on the reader) and the surgical margin (Figures 1 and 2). Specimen mammography was subsequently performed after excision to confirm the retrieval and position of the marker (Figures 3 and 4).

Endpoints. The primary endpoint was to assess the extent of successful Magseed/RFID tag deployment and retrieval. Secondary endpoints encompassed: Radial surgical margins, the need for re-operation, the weight of the surgical specimen in addition to the retrieval, migration rate $(>5 \mathrm{~mm})$ of the seed or tag, and the perspectives of patients, radiologists and the surgeon on the experience of using Magseeds/RFID tags compared to WGL. After the initial learning curve, the duration of Magseed/RFID tag deployment/surgical excision was recorded in the last seven cases of the series.

\section{Results}

The mean patient age was 56 years (range $=26-77$ years). Results are illustrated in Table I.

Disease characteristics. As expected, most lesions were the result of screen-detected non-palpable malignancy $(n=11 / 22)$. Nine of these patients had invasive malignancy and two had DCIS. The remaining lesions were malignant and rendered non-palpable following NACT $(n=6 / 22)$, three of which achieved a pathological complete response on final pathology, or preoperatively indeterminate non-palpable lesions which required surgical excision for diagnostic purposes $(n=5 / 22$; final pathology confirmed the diagnosis of one papilloma and two complex sclerosing lesions, one fibroadenoma and one lobular carcinoma in situ/atypical lobular hyperplasia). The final pathology details are shown in Table I.

Deployment, retrieval and migration. All patients experienced successful deployment and retrieval of both Magseeds and RFID tags (100\% and 100\%, respectively). The mean time required to deploy the Magseed/RFID tag under ultrasound guidance was $3.4 \mathrm{~min}$ (median of $3.5 \mathrm{~min}$, range $=2-5 \mathrm{~min}$ ). The mean time taken to identify and surgically excise the lesion using Magseed/RFID tag retrieval was $8.8 \mathrm{~min}$ (median of $8.5 \mathrm{~min}$, range $=6-15 \mathrm{~min}$ ). The recorded operating times excluded haemostasis, wound repair, and closure.

In all 22 cases, the mean distance between Magseed/RFID tag and the target lesion was $1.1 \mathrm{~mm}$ (median=0 mm, range $=0-10 \mathrm{~mm}$ ). Migration of the marker was observed in one case where the Magseed migrated by $1 \mathrm{~cm}$ due to the presence of haematoma caused by the stereotactic vacuum biopsy, yielding a mean migration rate of $4.5 \%$ overall. The depth of lesions from the surface of the skin was determined using preoperative imaging and ranged from 5 to $36 \mathrm{~mm}$ as measured. The deepest lesion was excised using RFID tag and LOCalizer (36 mm). 


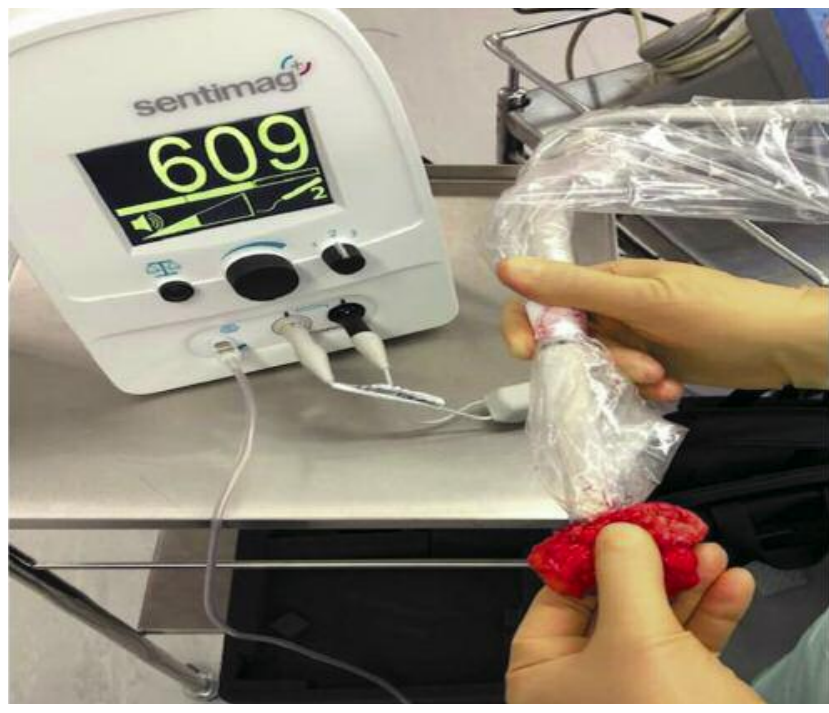

Figure 1. The use of a magnetic probe to assess proximity to the Magseed.

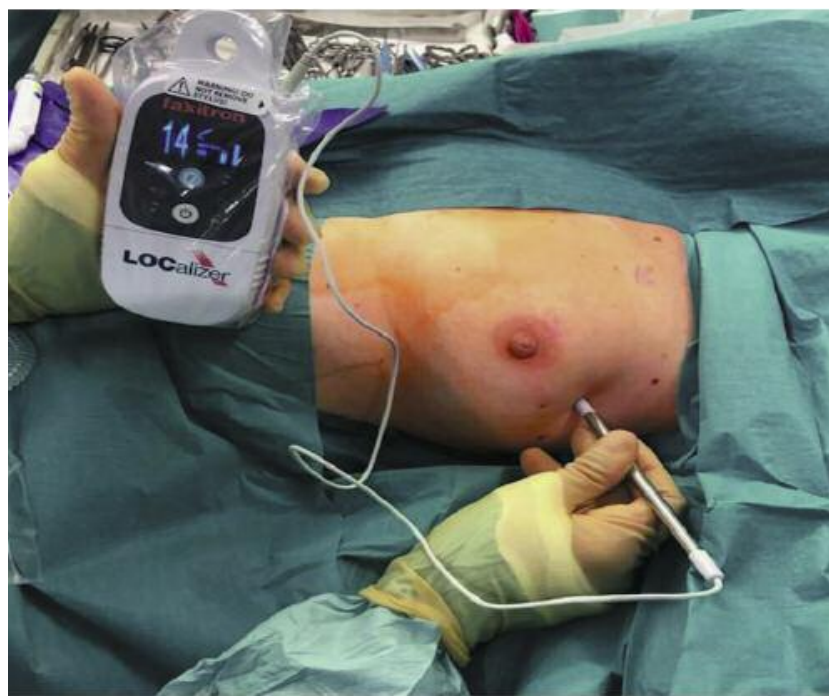

Figure 2. The use of a LOCalizer device to assess the distance in millimetres between the radiofrequency identification tag and the surgical margin after excision.

Surgical margins and specimen weight. Twenty-one cases (95\%) had clear surgical margins ( $\geq 2 \mathrm{~mm}$ for DCIS and $\geq 1$ $\mathrm{mm}$ for other lesions). For malignancy, the margin positivity rate was $5.9 \%$. Only one patient had positive surgical margins because the size and multifocality of the invasive lobular carcinoma had been underestimated by the preoperative magnetic resonance imaging (MRI). Following

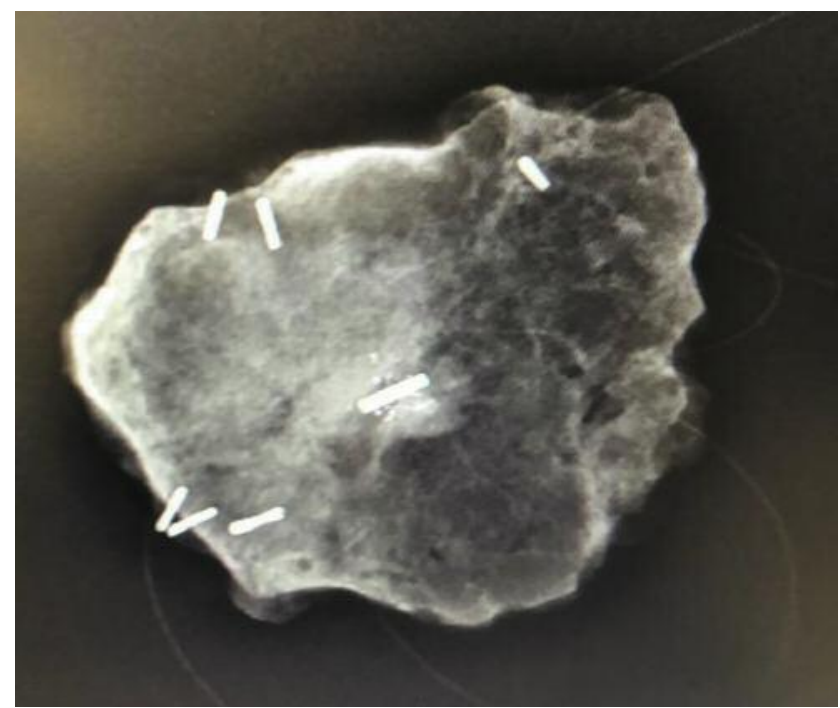

Figure 3. The use of a LOCalizer device to assess the distance in millimetres between the radiofrequency identification tag and the surgical margin after excision.

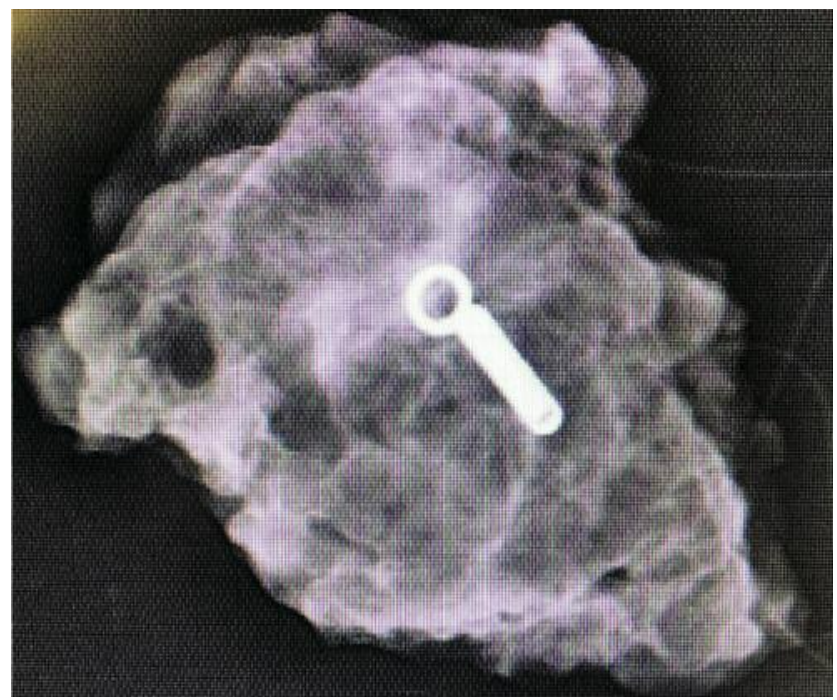

Figure 4. A specimen radiograph demonstrating the radiofrequency identification tag in the centre of the tumour.

repeat surgical excision, clear surgical margins were achieved for this patient. No patients experienced any pre-, intra- or post-operative complications.

The mean weight of resected specimens for malignancy was $16.7 \mathrm{~g}$ (median=10.5, range=6.6-62 g). Based on historical controls, this was lower than the expected weight for WGL for malignancy. The correlation between margin 
Table I. Pathological characteristics and localisation details.

\begin{tabular}{|c|c|c|c|c|c|c|}
\hline Case & $\begin{array}{l}\text { Age } \\
\text { (years) }\end{array}$ & $\begin{array}{l}\text { Distance of seed } \\
\text { to target }(\mathrm{mm})^{*}\end{array}$ & Localisation & $\begin{array}{l}\text { Specimen } \\
\text { weight }(\mathrm{g})\end{array}$ & $\begin{array}{c}\text { Pathology/pathological } \\
\text { TNM stage }\end{array}$ & Margins \\
\hline \multirow[t]{2}{*}{1} & 44 Lesion 1 & 1.9 & Magseed & 7.3 & ypTONO & Clear \\
\hline & Lesion 2 & 3.3 & Magseed & 7.6 & ypTONO & Clear \\
\hline 2 & 47 & 2.8 & Magseed & 62 & ypTisNO & Clear \\
\hline 3 & 49 & 1 & Magseed & 10.5 & урT0NO & Clear \\
\hline 4 & 75 & 0 & Magseed & 37.5 & T1N0 & Clear \\
\hline 5 & 56 & 10 & Magseed & 19 & pTis & Clear \\
\hline 6 & 26 & 0 & Magseed & 2.9 & Papilloma & Clear \\
\hline 7 & 77 & 0 & Magseed & 10.5 & pT1bN0 & Clear \\
\hline 8 & 48 & 0 & Magseed & 18 & ypTisNo & Clear \\
\hline 9 & 75 & 0 & Magseed & 14 & pT1bN0 & Clear \\
\hline 10 & 47 & 0 & Magseed & 11.8 & CSL & Clear \\
\hline 11 & 57 & 1 & Magseed & 7 & pT2N1 & Positive $(0.5 \mathrm{~mm})$ \\
\hline 12 & 65 & 0 & Magseed & 6.6 & pT1bN0 & Clear \\
\hline 13 & 53 & 0 & Magseed & 12.7 & pT1bN0 & Clear \\
\hline 14 & 61 & 0 & Localizer & 9 & ypTisNO & Clear \\
\hline 15 & 68 & 0 & Magseed & 7 & $\mathrm{pT} 1 \mathrm{cN} 0$ & Clear \\
\hline 16 & 43 & 0 & Localizer & 12.5 & CSL & Clear \\
\hline \multirow[t]{2}{*}{17} & 68 Lesion 1 & 0 & Localizer & 9.5 & pT1bN0 & Clear \\
\hline & Lesion 2 & 0 & Magseed & 17.5 & LCIS/ALH & Clear \\
\hline 18 & 62 & 2 & Localizer & 17 & pTis & Clear \\
\hline \multirow[t]{2}{*}{19} & 48 Lesion 1 & 0 & Localizer & 28 & pT1cN0 & Clear \\
\hline & Lesion 2 & 0 & Localizer & 3.5 & Fibroadenoma & Clear \\
\hline
\end{tabular}

CSL: Complex sclerosing lesion; LCIS: lobular carcinoma in situ; ALH: atypical lobular hyperplasia. *By mammography.

width and pathological margins, to a degree of $1 \mathrm{~mm}$, was $100 \%$ for the four tumours surgically resected using the LOCalizer.

Surgical and radiological perspectives. Both the surgeon and radiologists rated both techniques as being much better than wire localisation. The mean time for imaging-guided deployment was $3.4 \mathrm{~min}$ (median $=3.5$, range $=2-5 \mathrm{~min}$ ). The mean duration for surgical resection was $8.8 \mathrm{~min}$ (median $=8.5$, range $=6-15 \mathrm{~min}$ ).

The radiologists preferred Magseed due to the smaller deployment needle (18-gauge) and smaller seed size $(5 \mathrm{~mm})$. The 12-gauge introducer needle for LOCalizer was more difficult to use especially in dense breasts and potentially increased the risk of haematoma formation and tag migration through the needle track.

However, the surgeon preferred the LOCalizer, commenting that in addition to being lighter and easier to handle than the Sentimag probe, the LOCalizer facilitated more precise navigation and accurate estimation of distance from the probe to the tag. The need to remove metal instruments from the surgical field was identified as an intraoperative disadvantage for use of the Magseed technology.

Patient perspectives. Patient satisfaction data were recorded using a linear visual analogue scale $(n=10 / 19)$. The mean score was 9.7/10 (range=8-10). Two patients (one Magseed and one RFID) had previously undergone wire localisation and rated the new techniques as much better than that with wires.

\section{Discussion}

To our knowledge, this is the first report to prospectively evaluate the effectiveness of two wireless radiation-free localisation techniques (Magseed and LOCalizer) in the same clinical practice. Both technologies are Food and Drug Administration-approved and Council of Europe-marked. All patients underwent successful deployment and retrieval marker, with an overall migration rate of $4.5 \%$. Furthermore, radiologists, patients, and the surgeon considered Magseed and LOCalizer superior to WGL. Our observations are consistent with recent studies evaluating radiation-free non wire-localisation techniques in terms of successful placement, identification, migration and retrieval of marker $(6,8-10,15-20)$. Such studies have demonstrated that these techniques are non-inferior to WGL while overcoming many of the limitations of the latter. The Savi Scout device that utilizes infrared and electromagnetic principles has been used in over 5,000 procedures in the USA (8), whereas Magseed has been used in over 30,000 procedures. The LOCalizer was approved by the Food and Drug Administration more recently and its use is currently on the rise. 
The mean time for successful deployment in our study was $3.4 \mathrm{~min}$, which was significantly shorter than that reported for wire localisation (10 $\mathrm{min})(21)$ thus resulting in time efficiency in the radiology suite, with potential costeffectiveness implications.

The mean specimen weight of the excised malignant lesions in our analysis was $16.7 \mathrm{~g}$, which was lower than that reported in a similar cohort (9). Moreover, the margin positivity and reoperation rates observed in our study $(5.9 \%$ for malignant lesions) were lower than those reported for WGL $(13-21 \%)(7,8)$. The real-time constant navigation facilitates centring of the lesion within the resection specimen thus leading potentially to a smaller volume of resected tissue and a higher probability of achieving clear surgical margins.

Although our observations can be primarily attributed to more precise radiological deployment and surgical excision in a high-volume academic breast institution in addition to early tumour stage of screen-detected malignancy, other potential contributing factors include the administration of NACT, which resulted in pathological complete response in three cases, the use of cavity shavings and local practice guidelines regarding the definition of clear surgical margins. Other investigators reported no significant difference in positive margins and reoperation rates, volume of resection specimens or operating time between RFID localisation $(n=147)$ and WGL ( $\mathrm{n}=356$ ) (10). Similarly, no significant difference was observed between Magseed or reflector-guided localisation and WGL in terms of resection margins and specimen weight $(9,12)$. It is worth noting, however, none of these studies was a randomized controlled trial, thus limiting the robustness of their conclusions. The procedures in our series were performed by dedicated breast radiologists and a senior breast surgeon at an academic breast care facility and our results are therefore not generalizable to other institutions. Other limitations of our study include the small sample size and lack of a direct comparison with WGL.

Unlike Savi Scout, magnetic seeds and RFID technology create signal void artefacts that may limit the diagnostic accuracy of breast MRI when the marker is in place (8). The MRI phantom is approximately $2 \mathrm{~cm}$ for the RFID tag and 4 $\mathrm{cm}$ for the Magseed, but ongoing technical developments aim to reduce the signal void artefacts of these technologies on MRI. Furthermore, there is a need to compare these techniques at various breast depths since lesions deeper in the breast have been linked to increased difficulty in detecting signals (5).

The current licence in the European Union permits deployment of these markers within 30 days of surgery, however, this period has been extended to 1 year in the United States, thus permitting deployment at the time of diagnostic core biopsy in patients requiring NACT. However, if MRI is deemed to be the optimal imaging modality for monitoring response to treatment then the use of alternative techniques that lack any significant MRI artefacts such as the Savi Scout system would be preferable (4). Contrastenhanced mammography could be considered as an effective alternative to MRI in monitoring response to NACT when Magseeds or RFID tags are used (22).

Magseeds and RFID tags were used simultaneously in one patient in our series to remove two lesions, which avoided signal overlap. This principle can be also used for bracketing large DCIS lesions. Furthermore, Magseed and RFID tags can be deployed at the time of the diagnostic biopsy, which reduces the need for another procedure to place a marker clip prior to neoadjuvant chemotherapy. The 5-mm Magseed can also be used to mark a biopsy-proven lymph node prior to neoadjuvant chemotherapy in order to facilitate targeted axillary dissection (23). In this context, ultrasonography rather than MRI can be used to monitor the radiological response of clipped pathological lymph nodes to NACT (24). In contrast, the 10.5-mm RFID tag is not currently licensed to be used for this purpose due to its larger size (8). This limitation also applies to the Scout device, which measures $12 \mathrm{~mm}$ in diameter, despite the fact that the Food and Drug Administration recently expanded the indication for the Scout device to include soft tissue localisation such as lymph nodes. Hologic Inc. is currently refining its RFID tag to reduce its size in order to obtain a license for placement in lymph nodes. Magseeds can be also combined with superparamagnetic iron oxide nanoparticles used for sentinel node identification through a dorsal injection, and this appears to amplify the transcutaneous magnetic signal emanating from the tumour (12). However, superparamagnetic iron oxide has also been linked to artefacts and subsequently impaired MRI and should be used with caution in combination with Magseeds (13).

A distinct advantage of Magseed, RFID tag and Savi Scout localisation is the short learning curve and our report describes our initial experience. We also recorded faster radiological deployment of markers and shorter operating time compared with historical controls. In order to implement these new procedures globally, it is important to demonstrate cost-effectiveness in addition to oncological non-inferiority compared with the current standard of care, namely WGL. Although the initial purchase cost for the RFID tag or Magseed is higher than that for a guidewire, however, it is likely that the improved time efficiency in both the radiology suite and the operating room will lead to cost savings that can easily offset the apparent difference in cost. Furthermore, these markers can be deployed in suitable patients at the time of diagnostic biopsy thus avoiding a second localisation procedure with further reduction in cost. A recent study demonstrated that radioactive seedlocalisation was more cost-effective than WGL, and these savings may be comparable for Magseed and RFID (11). Moreover, specimen orientation with metal clips might be unnecessary since the distance to the target can be measured 
by the probe, resulting in further cost savings, provided that the marker is accurately deployed in the centre of the target lesion as demonstrated in Figure 4. This specimen radiograph demonstrates the RFID tag (a $10.5 \mathrm{~mm}$ rod) in contact with a ring metallic marker which was deployed at the time of diagnostic biopsy prior to neoadjuvant chemotherapy. The final pathology revealed residual DCIS which was excised by at least $2 \mathrm{~mm}$ (radial margins). Metallic clips were not used to orientate the specimen as the LOCalizer was used to assess the distance between the surgical margins and the tag. Additional savings can result from the potential omission of specimen radiography, especially with the use of the LOCalizer which can accurately estimate the distance between the specimen margin and the RFID tag.

These evolving technologies are likely to replace WGL in the near future in view of some clear advantages over WGL and preference by radiologists, surgeons and patients. No single wireless technique is perfect for all clinical scenarios and each method has certain advantages and disadvantages. High-volume breast surgery centres may consider having access to all three technologies and select the best technique for each patient. Technical failure seems to be rare with RFID tags and Magseeds, however, a failure rate of $3 \%$ has been reported for the Savi Scout device and this has been attributed to excessive depth of the target lesion, obscuration by a hematoma, or faulty reflectors (25). Alternative methods can be used in such cases of technical failure.

Future technological research should focus on the development of MRI-compatible introducer needles and the potential addition of energy to these markers to locally ablate tumours. Quantitative cost-effectiveness analysis and potential improvement of the aesthetic outcome should be included in future studies.

\section{Conclusion}

Our study further supports the concept that non-wire radiation-free localisation methods are effective and reliable alternatives to WGL, with time efficiency improvement in both the radiology suite and operating room. Magseed and RFID tags are novel tools which facilitate accurate surgical excision of non-palpable breast lesions and are associated with acceptably low margin positivity rates and high acceptance by patients, radiologists and surgeons.

\section{Conflicts of Interest}

None of the Authors has declared any financial conflict of interest. Both manufacturers provided equipment and technology support to the London Breast Institute in order to facilitate the clinical evaluation.

\section{Authors' Contributions}

$\mathrm{KM}$ initiated, designed and supervised the study, and proofread and finalised the article. ST and RG performed the literature review and drafted the manuscript. NP, MM and AM contributed to the article and design of database and deployment of markers.

\section{Acknowledgements}

This study was funded by a grant from the Breast Cancer Hope Foundation (London, UK).

\section{References}

1 DeSantis CE, Fedewa SA, Goding Sauer A, Kramer JL, Smith RA and Jemal A: Breast cancer statistics, 2015: Convergence of incidence rates between Black and White women. CA Cancer J Clin 66(1): 31-42, 2016. PMID: 26513636. DOI: 10.3322/caac. 21320

2 Park H-L and Kim LS: The current role of vacuum-assisted breast biopsy system in breast disease. J Breast Cancer 14(1): 17, 2011. PMID: 21847387. DOI: 10.4048/jbc.2011.14.1.1

3 Gray RJ, Salud C, Nguyen K, Dauway E, Friedland J, Berman C, Peltz E, Whitehead G and Cox CE: Randomized prospective evaluation of a novel technique for biopsy or lumpectomy of nonpalpable breast lesions: Radioactive seed wire localization. Ann Surg Oncol 8(9): 711-715, 2001. PMID: 11597011. DOI: 10.1007/s10434-001-0711-3

4 Mango VL, Wynn RT, Feldman S, Friedlander L, Desperito E, Patel SN, Gomberawalla A and Ha R: Beyond wires and seeds: Reflector-guided breast lesion localization and excision. Radiology 284(2): 365-371, 2017. PMID: 28430555. DOI: 10.1148/radiol.2017161661

5 Harvey JR, Lim Y, Murphy J, Howe M, Morris J, Goyal A and Maxwell AJ: Safety and feasibility of breast lesion localization using magnetic seeds (Magseed): a multi-centre, open-label cohort study. Breast Cancer Res Treat 169(3): 531-536, 2018. PMID: 29453521. DOI: 10.1007/s10549-018-4709-y

6 Dauphine C, Reicher JJ, Reicher MA, Gondusky C, Khalkhali I and Kim MA: Prospective clinical study to evaluate the safety and performance of wireless localization of nonpalpable breast lesions using radiofrequency identification technology. Am J Roentgenol 204(6): W720-723, 2015. PMID: 26001262. DOI: 10.2214/AJR.14.13201

7 Corsi F, Sorrentino L, Bossi D, Sartani A and Foschi D: Preoperative localization and surgical margins in conservative breast surgery. Int J Surg Oncol 2013: 793819, 2013. PMID: 23986868. DOI: $10.1155 / 2013 / 793819$

8 Hayes MK: Update on preoperative breast localization. Radiol Clin North Am 55: 591-603, 2017. PMID: 28411682. DOI: 10.1016/j.rcl.2016.12.012

9 Zacharioudakis K, Down S, Bholah Z, Lee S, Khan T, Maxwell AJ, Howe M and Harvey J: Is the future magnetic? Magseed localisation for non palpable breast cancer. A multi-centre non randomised control study. Eur J Surg Oncol 45(11): 2016-2021, 2019. PMID: 31288944. DOI: 10.1016/j.ejso.2019.06.035.

10 McGugin C, Spivey T, Coopey S, Smith B, Kelly B, Gadd M, Hughes K, Dontchos B and Specht M: Radiofrequency identification tag localization is comparable to wire localization 
for non-palpable breast lesions. Breast Cancer Res Treat 177(3): 735-739, 2019. PMID: 31302856. DOI: 10.1007/s10549-01905355-0

11 Zhang Y, Seely J, Cordeiro E, Hefler J, Thavorn K, Mahajan M, Domina S, Aro J, Ibrahim AM, Arnaout A, Gravel D and Nessim $\mathrm{C}$ : Radioactive seed localization versus wire-guided localization for nonpalpable breast cancer: a cost and operating room efficiency analysis. Ann Surg Oncol 24(12): 3567-3573, 2017. PMID: 28913761. DOI: 10.1245/s10434-017-6084-Z

12 Hersi AF, Eriksson S, Ramos J, Abdsaleh S, Wärnberg F and Karakatsanis A: A combined, totally magnetic technique with a magnetic marker for non-palpable tumour localization and superparamagnetic iron oxide nanoparticles for sentinel lymph node detection in breast cancer surgery. Eur J Surg Oncol 45(4): 544-549, 2019. PMID: 30401506. DOI: 10.1016/j.ejso.2018.10.064

13 Krischer B, Forte S, Niemann T, Kubik-Huch RA and Leo C: Feasibility of breast MRI after sentinel procedure for breast cancer with superparamagnetic tracers. Eur J Surg Oncol 44(1): 74-79, 2018. PMID: 29217399. DOI: 10.1016/j.ejso.2017.11.016

14 Schermers B van der Hage JA, Loo CE, Vrancken Peeters MTFD, Winter-Warnars HAO, van Duijnhoven F, Ten Haken B, Muller SH and Ruers TJM: Feasibility of magnetic marker localisation for non-palpable breast cancer. Breast 33: 50-56, 2017. PMID: 28282587. DOI: 10.1016/j.breast.2017.03.003

15 Price ER, Khoury AL, Esserman LJ, Joe BN and Alvarado MD: Initial clinical experience with an inducible magnetic seed system for preoperative breast lesion localization. Am J Roentgenol 210(4): 913-917, 2018. PMID: 29446680. DOI: 10.2214/AJR.17.18345

16 Lamb LR, Bahl M, Specht MC, D’Alessandro HA and Lehman $\mathrm{CD}$ : Evaluation of a nonradioactive magnetic marker wireless localization program. Am J Roentgenol 211(4): 940-945, 2018. PMID: 30085842. DOI: 10.2214/AJR.18.19637

17 DiNome ML, Kusske AM, Attai DJ, Fischer CP and Hoyt AC: Microchipping the breast: an effective new technology for localizing non-palpable breast lesions for surgery. Breast Cancer Res Treat 175(1): 165-170, 2019. PMID: 30689105. DOI: 10.1007/s10549-019-05143-w

18 Jadeja PH, Mango V, Patel S, Friedlander L, Desperito E, AyalaBustamante E, Wynn R, Chen-Seetoo M, Taback B, Feldman S and $\mathrm{Ha}$ R: Utilization of multiple SAVI SCOUT surgical guidance system reflectors in the same breast: a single-institution feasibility study. Breast J 24(4): 531-534, 2018. PMID: 29251387. DOI: $10.1111 /$ tbj.12979
19 Patel SN, Mango VL, Jadeja P, Friedlander L, Desperito E, Wynn R, Feldman S and Ha R: Reflector-guided breast tumor localization versus wire localization for lumpectomies: A comparison of surgical outcomes. Clin Imaging 47: 14-17, 2018. PMID: 28818762. DOI: 10.1016/j.clinimag.2017.07.020

20 Cox CE, Garcia-Henriquez N, Glancy MJ, Whitworth P, Cox JM, Themar-Geck M, Prati R, Jung M, Russell S, Appleton K, King $\mathbf{J}$ and Shivers SC: Pilot study of a new nonradioactive surgical guidance technology for locating nonpalpable breast lesions. Ann Surg Oncol 23(6): 1824-1830, 2016. PMID: 26847680. DOI: $10.1245 / \mathrm{s} 10434-015-5079-\mathrm{x}$

21 Tardioli S, Ballesio L, Gigli S, DI Pastena F, D’Orazi V, Giraldi G, Monti M, Amabile MI and Pasta V: Wire-guided localization in non-palpable breast cancer: Results from monocentric experience. Anticancer Res 36(5): 2423-2427, 2016. PMID: 27127152.

22 Barra FR, Sobrinho AB, Barra RR, Magalhães MT, Aguiar LR, de Albuquerque GFL, Costa RP, Farage L and Pratesi R: Contrastenhanced mammography (CEM) for detecting residual disease after neoadjuvant chemotherapy: A comparison with breast magnetic resonance imaging (MRI). Biomed Res Int 2018: 8531916, 2018. PMID: 30533440. DOI: $10.1155 / 2018 / 8531916$

23 Greenwood HI, Wong JM, Mukhtar RA, Alvarado MD and Price ER: Feasibility of magnetic seeds for preoperative localization of axillary lymph nodes in breast cancer treatment. Am J Roentgenol 213(4): 953-957, 2019 . PMID: 31166765. DOI: 10.2214/AJR.19.21378

24 Candelaria RP, Bassett RL, Symmans WF, Ramineni M, Moulder SL, Kuerer HM, Thompson AM and Yang WT: Performance of mid-treatment breast ultrasound and axillary ultrasound in predicting response to neoadjuvant chemotherapy by breast cancer subtype. Oncologist 22(4): 394-401, 2017. PMID: 28314842. DOI: 10.1634/theoncologist.2016-0307

25 Falcon S, Weinfurtner RJ, Mooney B and Niell BL: SAVI SCOUT $^{\circledR}$ localization of breast lesions as a practical alternative to wires: Outcomes and suggestions for trouble-shooting. Clin Imaging 52: 280-286, 2018. PMID: 30193186. DOI: 10.1016/j.clinimag.2018.07.008

Received November 1, 2019

Revised November 20, 2019

Accepted November 25, 2019 